\title{
Bradykinin receptors and EphB2/EphrinB2 pathway in response to high glucose-induced osteoblast dysfunction and hyperglycemia-induced bone deterioration in mice
}

\author{
MIN WU ${ }^{1,2}$, WENTING AI ${ }^{3}$, LIN CHEN ${ }^{4}$, SIHAI ZHAO ${ }^{1}$ and ENQI LIU ${ }^{1}$ \\ ${ }^{1}$ Laboratory Animal Center, Xi'an Jiaotong University School of Medicine, Xi'an, Shaanxi 710061; \\ Departments of ${ }^{2}$ Endocrinology, ${ }^{3}$ Cardiology and ${ }^{4}$ Pathology, Shaanxi Provincial \\ People's Hospital, Xi'an, Shaanxi 710068, P.R. China
}

Received July 20, 2015; Accepted December 30, 2015

DOI: $10.3892 /$ ijmm.2016.2457

\begin{abstract}
This study was carried out in order to investigate bone dysfunction and the involvement of bradykinin receptors and the Eph/Ephrin signaling pathway in osteoblasts and in mice with diabetes-related osteoporosis in response to exposure to high glucose. Osteogenic transdifferentiation was inhibited when the osteoblasts were exposed to high glucose, and the expression levels of bone formation-related genes [Runx 2 and alkaline phosphatase (ALP)] were decreased, while those of bone resorption-related genes [matrix metalloproteinase (MMP)9 and carbonic anhydrase II (CAII)] were increased. Moreover, the mRNA and protein expression levels of bradykinin receptor B1 (BK1R)/bradykinin receptor B2 (BK2R) and EphB2/EphrinB2 were significantly decreased in the osteoblasts following exposure to high glucose. Intriguingly, the interaction between BK2R and EphB2/EphrinB2 was confirmed, and BK2R loss-of-function significantly decreased the mRNA and protein expression levels of EphB2/EphrinB4. In vivo, hyperglycemia induced the disequilibrium of calcium homeostasis through the inhibition of bone formation and the acceleration of bone resorption, which was manifested by the reduction of trabecular bone mass of the primary and secondary spongiosa, as well as by the increase in the number of mature osteoclasts throughout the proximal tibial metaphysis in mice with diabetes-related osteoporosis. Furthermore, the mRNA and protein expression levels of BK1R/BK2R and EphB2/EphrinB2 in the tibias of the mice with diabetes-related osteoporosis were significantly decreased. These results demonstrate that bradykinin receptors and the EphB4/EphrinB2 pathway mediate the development of
\end{abstract}

Correspondence to: Dr Enqi Liu, Laboratory Animal Center, Xi'an Jiaotong University School of Medicine, 76 West Yanta Road, Xi'an, Shaanxi 710061, P.R. China

E-mail: medicineleq@outlook.com

Key words: Eph/Ephrin, bradykinin receptor, hyperglycemia, bone complications in mice with diabetes-related osteoporosis and suggest that the inactivation of bradykinin receptors and the EphB4/EphrinB2 pathway enhance the severity of complications in mice with diabetes-related osteoporosis.

\section{Introduction}

Osteoporosis and falls are related to fractures, which can lead to increased morbidity and mortality, as well as decreased functional ability. The mortality of patients with hip fractures within 1 year is $20 \%$, and only one-third of these patients recover their original functions (1). Diabetes mellitus (DM) is an endocrine metabolic dysfunctional disease, which is frequently accompanied by osteoporosis (2). DM results in hyperglycemia due to an absolute insulin insufficiency or insulin resistance, which leads to the development of a number of complications, both microvascular and macrovascular pathological changes (3). Clinical surveys have indicated that DM enhances the risk of hip fractures, vertebral fractures, proximal humeral fractures, tibia fractures, and wrist and ankle fractures, independent of bone mineral density (BMD) $(4,5)$. Moreover, DM has been shown to be associated with the delayed healing of fractures and bone deteriorations in animal models $(6,7)$. In the KK-Ay mouse, a classic animal model of obesity with T2DM, high insulin levels increased cortical bone mass and impaired the trabecular microstructure by upregulating osteoblast-and osteoclastrelated gene expression (8). In vitro, high glucose $(16.5 \mathrm{mM})$ has been shown to inhibit the osteogenic differentiation potential and proliferation of rat osteoblasts; moreover, high glucose significantly impairs bone formation by inhibiting osteoblast proliferation and differentiation $(9,10)$. Although the in vitro and in vivo contribution of glucose to bone metabolism has been investigated, the signaling mechanisms responsible for high glucose-induced bone deteriorations have not yet been well characterized.

Bone is remodeled constantly throughout life by boneresorbing osteoclasts and bone-forming osteoblasts. To maintain bone volume and quality, the differentiation of osteoclasts and osteoblasts is tightly regulated through communication between and within these two cell lineages (11). Bone cells, such as chondrocytes, osteoblasts, osteocytes and osteoclasts, 
express a variety of Ephrin ligands and Eph receptors $(12,13)$. Cultured osteoclasts stimulated with receptor activator of nuclear factor- $\kappa \mathrm{B}$ ligand (RANKL) have been shown to dynamically express Ephrin ligands (A2, B1 and B2) and Eph receptors (A1, A2 and A4), as revealed by reverse transcriptionpolymerase chain reaction (RT-PCR) $(11,14)$. The protein expression of Ephrin ligands and Eph receptors was also identified in human mesenchymal stem cells (MSCs) by western blot analysis and immunohistochemistry (15). Developmental deficiencies in the EphB/EphrinB signaling pathway can lead to skeletal abnormalities, which includes the defective development of the somite (16), craniofacial development (17), limb development (18), and other bone abnormalities in mutant mice, and in individuals harboring EphrinB1 mutations that cause the X-linked developmental disorder craniofrontonasal syndrome (19). The osteoclasts of EphA4 null mice are also larger, and express higher mRNA levels of matrix metalloproteinase (MMP)3 and MMP9, and exhibit greater bone resorption activity than wild-type osteoclasts in vitro (20). A microarray analysis of bone marrow-derived MSCs (BMSCs) identified EphA5 as an inhibitory factor candidate, which may be a negative regulator of bone formation (21). Moreover, cell-cell interactions mediated by the EphrinB2 ligand on osteoclasts have been shown to inhibit osteoclast-induced bone resorption, and the EphB4 receptor on osteoblasts can promote osteoblast-driven bone formation (22). By contrast, bidirectional EphrinA2/EphA2 signaling regulates bone remodeling at the initiation phase through the enhancement of osteoclastogenesis and the suppression osteoblastogenesis (11). Although the effects of the Eph/Ephrin signaling pathway can lead to skeletal abnormalities, the role of Eph/Ephrin in the process of bone deteriorations induced by hyperglycemia remains largely unknown.

Previous studies have shown that kininogen deficiency and bradykinin receptor loss-of-function provide evidence of an important role of kinin peptides in the regulation of blood pressure and sodium homeostasis, renal responses to vasopressin and insulin sensitivity in rodents $(23,24)$. Bradykinin receptor B2 (BK2R) gene knockout can induce cardiomyopathy in mice, and mice with tissue kallikrein gene knockout develop impaired flow-induced vasodilatation (24). In mice, the knockdown of bradykinin receptor B1 (BK1R; BK1R ${ }^{-/-}$mice), has been shown to result in increased bone loss; bone marrow cells obtained from BK1R $\mathrm{R}^{-/-}$mice displayed an enhanced differentiation ability into functional osteoclasts with consistent artificial calcium phosphate degradation (25). Intriguingly, the lack of BK1R and BK2R exacerbates diabetes-associated complications, such as renal injury and bone mineral loss, and bradykinin receptors play a protective role in diabetes-related osteoporosis (26).

The above-mentioned data suggest that Eph-Ephrin bidirectional signaling provides an intriguing explanation for the cellular and molecular mechanisms which are responsible for osteoblast-osteoclast coupling and may prove to be a potential target for the treatment of osteoporosis. Thus, the aim of the present study was to examine the involvement of the skeletal Eph/Ephrin signaling pathway and bradykinin receptors in bone deteriorations induced by hyperglycemia in vitro, as well as in vivo using an animal model of streptozotocin (STZ)induced diabetes.

\section{Materials and methods}

Culture of MC3T3 osteoblasts. MC3T3 osteoblasts were obtained from the Chinese Academy of Sciences (Institute of Shanghai Cell Biology and Chinese Type Culture Collection, Shanghai, China), and the cells were maintained in Dulbecco's modified Eagle's medium (DMEM; Invitrogen, Carlsbad, CA, USA), supplemented with $10 \%$ fetal bovine serum (FBS; HyClone, Logan, UT, USA), $100 \mu \mathrm{g} / \mathrm{ml}$ penicillin and $100 \mu \mathrm{g} / \mathrm{ml}$ streptomycin (Invitrogen) at $37^{\circ} \mathrm{C}$ in a humidified, $5 \% \mathrm{CO}_{2}, 95 \%$ air atmosphere. The medium was changed to fresh medium initially after $24 \mathrm{~h}$ and then every 2 days.

Animal treatment. Six-week-old male C57BL/6J mice (from the Animal Center of Xi'an Jiaotong University, Xi'an, China) were allowed to acclimatize to their environment for 1 week. All experimental procedures were carried out in accordance with the guidelines of Xi'an Jiaotong University on Animal Care. All chemicals and reagents were purchased from Sigma (Oakville, ON, Canada), unless otherwise stated. The mice were randomly divided into 2 groups as follows: i) the control group (Cont, $\mathrm{n}=10)$; ii) the STZ group ( $\mathrm{n}=10)$, where the animals were rendered hyperglycemic by an intraperitoneal injection of STZ, dissolved in citrate buffer ( $0.1 \mathrm{M}$ at $\mathrm{pH} 4.2)$, at $35 \mathrm{mg} / \mathrm{kg}$ body weight for 5 consecutive days. The mice in the control group was treated with citrate buffer only. All the mice were sacrificed by cardiac exsanguination under light ether anesthesia 8 weeks after the STZ injection. The fasting blood glucose (FBG) levels were measured using a blood glucose monitoring system (Roche Diagnostics, Manheim, Germany), and the body weight of the mice was recorded every 2 weeks during the experimental period.

\section{3-(4,5-Dimethylthiazol-2-yl)-2,5-diphenyltetrazolium bromide} (MTT) assay. The osteoblasts were cultured in 96-well plates (at $10^{3}$ cells/well). An MTT assay was conducted after 1, 4 or 7 days of exposure to high glucose $(16.5 \mathrm{mM})$ using a cell proliferation kit (Sigma, Oakville, ON, Canada) according to the manufacturer's instructions. After $24 \mathrm{~h}$, the culture medium was replaced with $100 \mathrm{ml}$ of MTT $(0.5 \mathrm{mg} / \mathrm{ml})$. The black crystals that formed after 2-3 $\mathrm{h}$ were dissolved with acidified isopropanol and the absorbance was measured at $570 \mathrm{~nm}$ using a microplate reader (MD SpectraMax M5; Molecular Devices, Sunnyvale, CA, USA).

Quantification of apoptosis by flow cytometry. Apoptosis was assessed using Annexin V, a protein that binds to phosphatidylserine (PS) residues which are exposed on the cell surface of apoptotic cells. The osteoblasts were treated with the vehicle (normal glucose; $5.5 \mathrm{mM}$ ) or high glucose for 4 days. Following treatment, the cells were washed twice with phosphate-buffered saline (PBS; $\mathrm{pH} 7.4$ ), and re-suspended in staining buffer containing $1 \mu \mathrm{g} / \mathrm{ml}$ propidium iodide (PI) and $0.025 \mu \mathrm{g} / \mathrm{ml}$ Annexin V-FITC. Double-labeling was performed at room temperature for $10 \mathrm{~min}$ in the dark prior to flow cytometric analysis. The osteoblasts were immediately analyzed using FACScan and the CellQuest program. The quantitative assessment of apoptotic cells was also assessed by the terminal deoxynucleotidyl transferase-mediated deoxyuridine triphosphate nick-end labeling (TUNEL) method, which examines DNA-strand breaks during apoptosis using a BD ApoAlert ${ }^{\mathrm{TM}}$ 
DNA Fragmentation assay kit. The stained cells were then analyzed using a flow cytometer (FC500; Beckman Coulter, Miami, FL, USA).

Concentrations of calcium $(\mathrm{Ca})$ and creatinine $(\mathrm{Cr})$ in serum and urine. The concentrations of $\mathrm{Ca}$ and $\mathrm{Cr}$ in the serum and urine of the mice were measured using standard colorimetric methods with a microplate reader (Bio-Tek, Winooski, VT, USA). Serum was collected by cardiac exsanguination under light ether anesthesia. After $24 \mathrm{~h}$ urine was collected by metabolic cages. The level of $\mathrm{Ca}$ in the urine was corrected by the concentration of urine $\mathrm{Cr}$ levels. The serum levels of tartrate-resistant acid phosphatase-5b (TRACP-5b) and osteocalcin (OCN) were detected using a mouse bioactive enzyme-linked immunosorbent assay (ELISA; Immutopics, Inc., San Clemente, CA, USA) with an ELISA reader (MD SpectraMax M5; Molecular Devices).

Gene silencing by small interfering RNA (siRNA). For siRNA experiments, RNA primers complementary to BK2R were designed and synthesized by the Shanghai Invitrogen Biotechnology Co. (Shanghai, China). The osteoblasts were transfected with the annealed RNA primer pair using Lipofectamine 2000 (Invitrogen) in accordance with the instructions provided by the manufacturer. Cells transfected with scrambled siRNA served as controls. The siRNA had the following sequences: BK2R siRNA, 5'-ACGGCCCACCAGUCGCCCCAG-3'; and scrambled siRNA, 5'-UUCGACGGUCAGCACGCGAGC-3'.

Mineralization assay. Mineralized matrix formation was assessed by Alizarin Red S staining and the Ca45 intake method. The osteoblasts were fixed in $4 \%$ paraformaldehyde and stained with $40 \mathrm{mmol} / \mathrm{l}$ Alizarin Red S (pH 4.2; SigmaAldrich, Munich, Germany) for $30 \mathrm{~min}$ at room temperature. The excess dye was removed by washing the plates with distilled water. The incorporated calcium was eluted with $100 \mathrm{mmol} / 1$ cetylpyridinium chloride and visualized under a microscope (Leica DM 2500; Leica Microsystems GmbH, Wetzlar, Germany).

Bone histomorphology. Following fixation in $4 \%$ formaldehyde solution for 7 days, the tibias were decalcified in $0.5 \mathrm{M}$ EDTA ( $\mathrm{pH} \mathrm{8.0)}$ and then embedded in paraffin using standard histological procedures. After cardiac exsanguination, the tibias were collected with all soft tissue removed and stored at $-80^{\circ} \mathrm{C}$. Sections $(4-\mu \mathrm{m}$-thick) were cut and stained with hematoxylin and eosin (H\&E), and visualized under a microscope (Leica DM 2500). TRAP staining was used for the identification of the osteoclasts according to the manufacturer's instructions (387-A; Sigma-Aldrich, St. Louis, CA, USA). The number of osteoclasts was quantified below the growth plates in the proximal tibial metaphysis, and visualized under a microscope (Leica DM 2500).

$R T-P C R$. RNA extraction was performed using TRIzol reagent according to the manufacturer's instructions (Invitrogen). cDNA synthesis was performed by reverse transcription reactions with $2 \mu \mathrm{g}$ of total RNA using moloney murine leukemia virus reverse transcriptase (Invitrogen) with oligo(dT)15 primers (Fermentas, Burlington, ON, Canada) as described by the manufacturer. First strand cDNA served as the template for the regular PCR performed using a DNA engine (ABI 7300). Glyceraldehyde 3-phosphate dehydrogenase (GAPDH) as an internal control was used to normalize the data to determine the relative expression levels of the target genes. The reaction conditions were set according to the instructions provided with the kit. The PCR primers used in this study were as follows: runt-related transcription factor (Runx2) forward, 5'-CCT GACTCTGCACCAAGTC-3' and reverse, 5'-GAGGTGGC AGTGTCATCATC-3'; alkaline phosphatase (ALP) forward, 5'-GCTGAACAGGAACAACGTGA-3' and reverse, 5'-AGA CTGCGCCTGGTAGTTGT-3'; MMP9 forward, 5'-GGTCGG TTCTGACCTTTTGT-3' and reverse, 5'-TGGTGTCCTCCGA TGTAAGA-3'; carbonic anhydrase II (CAII) forward, 5'-TGG TTCACTGGAACACCAAA-3' and reverse, 5'-AGCAAGGGT CGAAGTTAGCA-3'; osteoprotegerin (OPG) forward, 5'-TGC TCCTGGCACCTACCTA-3' and reverse, 5'-ACTCCTGC TTCACGGACTG-3'; RANKL forward, 5'-GGAAGCGTACC TACAGACTA-3' and reverse, 5'-AGTACGTCGCATCTT GATCC-3'; EphB4 forward, 5'-AGTGGCTTCGAGCCA TCAAGA-3' and reverse, 5'-CTCCTGGCTTAGCTTGGG ACTTC-3'; EphrinB2 forward, 5'-ACGGTCCAACAAG ACGTCCA-3' and reverse, 5'-GCTGTTGCCATCGGTG CTA-3'; BK1R forward, 5'-TGGAACCAGTTTAACTG GCC-3' and reverse, 5'-ATGAAGTCCTCCCAAAAGCA-3'; BK2R forward, 5'-TGTTCGTGAGGACTCCGTGC-3' and reverse, 5'-GCCAGGATCAGGTCTGCTG-3'; and GAPDH forward, 5'-CACCATGGAGAAGGCCGGGG-3' and reverse, 5'-GACGGACACATTGGGGGTAG-3'.

Immunoprecipitation and western blot analysis. Cell lysates were prepared as previously described (27). The lysates were then incubated with the indicated antibodies for $1 \mathrm{~h}$ at $4^{\circ} \mathrm{C}$. The immune complexes were precipitated with protein A/G agarose (Santa Cruz Biotechnology, Inc., Santa Cruz, CA, USA) for $1 \mathrm{~h}$ at $4^{\circ} \mathrm{C}$, washed extensively with lysis buffer, resolved in 4-20\% gradient sodium dodecyl sulfate-polyacrylamide gel electrophoresis (SDS-PAGE), and analyzed by western blotting. All blots were developed by enhanced chemiluminesence (Amersham Pharmacia Biotech, Piscataway, NJ, USA). The cells and tibias were homogenized and extracted in NP-40 buffer, followed by 5-10 min of boiling and centrifugation $\left(12,000 \mathrm{x} \mathrm{g}\right.$, for $15 \mathrm{~min}$ at $\left.4^{\circ} \mathrm{C}\right)$ to obtain the supernatant. Samples containing $60 \mu \mathrm{g}$ of protein were separated on a $10 \%$ SDS-PAGE gel and transferred onto nitrocellulose membranes (Bio-Rad Laboratories, Hercules, CA, USA). Following saturation with 5\% (w/v) non-fat dry milk in TBS and $0.1 \%(\mathrm{w} / \mathrm{v})$ Tween-20 (TBST), the membranes were incubated with the following antibodies: Bcl-2 (sc-7382), BAX (sc-20067), caspase-3 (sc-392736), caspase-8 (sc-81656), BK1R (sc-293196), BK2R (sc-136216), EphB4 (sc-130081) and EphrinB2 (sc-398735) (Santa Cruz Biotechnology, Inc.) at dilutions ranging from 1:500 to $1: 2,000$ at $4^{\circ} \mathrm{C}$ overnight. Following 3 washes with TBST, the membranes were incubated with secondary immunoglobulins (Igs; sc-2002) conjugated to IRDye 800CW Infrared Dye (LI-COR Biotechnology, Lincoln, NE, USA), including donkey anti-goat IgG and donkey antimouse IgG at a dilution of 1:10,000-1:20,000. Following $1 \mathrm{~h}$ of incubation at $37^{\circ} \mathrm{C}$, the membranes were washed 3 times with TBST. The blots were visualized using the Odyssey Infrared Imaging System (LI-COR Biotechnology). The signals were 


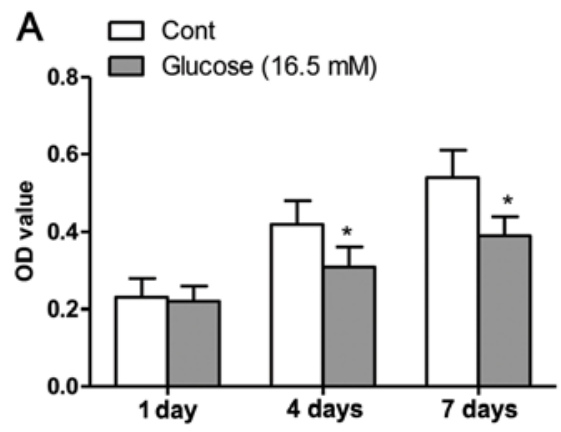

C

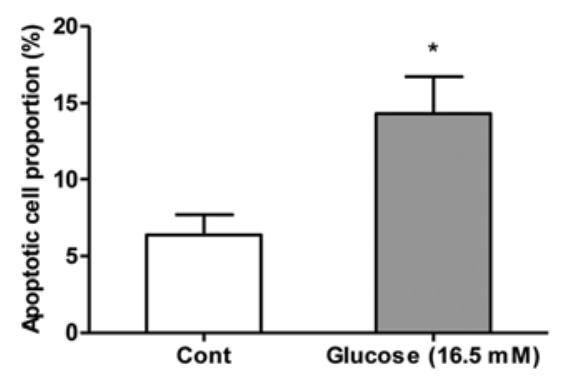

B
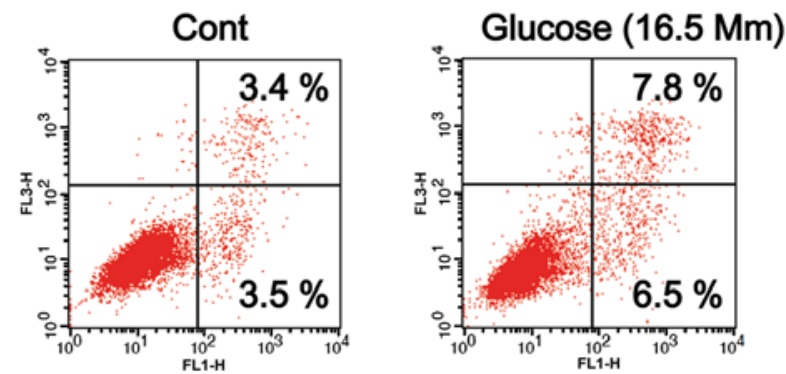

D

\section{Cont Glucose (16.5 mM)}

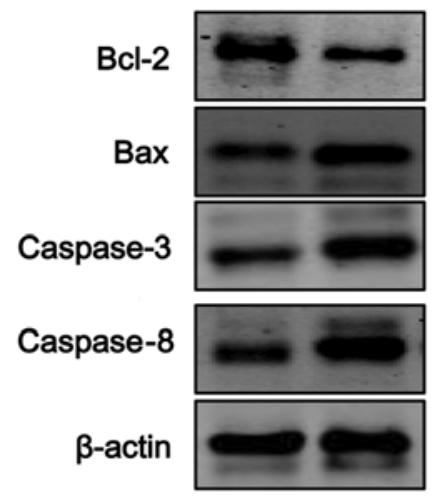

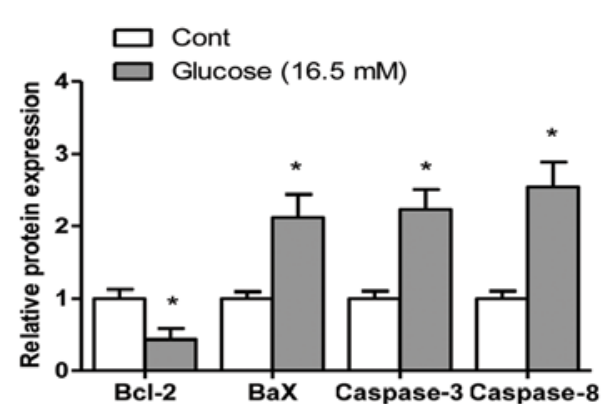

Figure 1. Exposure to high glucose induces osteoblast apoptosis. (A) Osteoblasts were incubated with high glucose (16.5 mM) for 1,4 or 7 days, and cell viability was examined by MTT assay. (B) Following exposure to high glucose (16.5 mM) for 4 days, the osteoblasts are subjected to flow cytometric analysis with Annexin V/propidium iodide (PI) double staining; upper right and lower right quadrants represent the proportion of late and early apoptotic cells, respectively. (C) The percentage of apoptotic cells was also determined. (D) The protein expression levels of Bcl-2, Bax, caspase-3 and caspase-8 were measured by western blot analysis when the cells were exposed to high glucose for 4 days. Values are expressed as the mean $\pm \mathrm{SEM}, \mathrm{n}=3$ parallel experiments in each group. ${ }^{*} \mathrm{P}<0.05$ vs. control group (normal glucose).

densitometrically assessed (Odyssey Application software version 3.0) and normalized to the mouse monoclonal anti- $\beta$ actin antibody (AP0733; Bioworld Technology, Inc., St. Louis Park, MN, USA).

Statistical analysis. The data from our experiments are reported as the means \pm standard errors of mean (SEM) for each group. All statistical analyses were performed using GraphPad Prism version 5.0 (GraphPad Software, La Jolla, CA, USA). Inter-group differences were analyzed by one-way ANOVA, followed by Tukey's multiple comparison test as a post-test to compare the group means if overall the P-value was $<0.05$. A value of $\mathrm{P}<0.05$ was considered to indicate a statistically significant difference.

\section{Results}

High glucose induces osteoblast apoptosis. In order to examine the detrimental effects of high glucose on osteoblasts, we exposed the osteoblasts to high glucose $(16.5 \mathrm{mM})$ for different periods of time. We first examined the viability of the osteoblasts after 1, 4 or 7 days of exposure to high glucose by MTT assay. The results revealed that exposure to high glucose induced osteoblast cell death in a time-dependent manner (Fig. 1A). To determine whether high glucose induced cell death through an apoptotic mechanism, Annexin V-PI double-labeling was used for the detection of the proportion of apoptotic osteoblasts. The apoptotic rate was significantly increased in the osteoblasts following exposure to high glucose for 4 days $(\mathrm{P}<0.05$; Fig. $1 \mathrm{~B}$ and $\mathrm{C})$. Finally, we measured the expression levels of apoptosis-related proteins. Exposure to high glucose significantly decreased the protein expression levels of Bcl-2 and increased the protein expression of Bax, caspase- 3 and caspase- 8 compared to the control cells (exposure to normal glucose) (Fig. 1D).

High glucose inhibits osteogenic differentiation. The mineralization capacity of the osteoblasts was assessed by Alizarin Red S staining (Fig. 2A), which stains deposited calcium red. Osteogenic transdifferentiation was inhibited when the osteoblasts were exposed to high glucose. Moreover, exposure of the cells to high glucose significantly decreased the mRNA expression levels of Runx2 and ALP, and increased the mRNA expression levels of MMP9 and CAII compared to the control cells (exposed to normal glucose) (Fig. 2B and C). The maturation and formation of osteoclasts are mainly regulated by the balance of extracellular OPG and RANKL, which are secreted by osteoblasts. Thus, in this study, we measured the expression levels of OPG and RANKL, and the ratio of OPG/RANKL in osteoblasts (28). The results from RT-PCR revealed that the mRNA expression levels of OPG and the ratio of OPG/RANKL were significantly decreased in the osteoblasts exposed to high glucose $(16.5 \mathrm{mM})$ compared to the control cells $(\mathrm{P}<0.05$; Fig. 2D). By contrast, the mRNA expression of RANKL was significantly higher in the cells exposed to high glucose compared to the control cells ( $\mathrm{P}<0.05$; Fig. 2D). 
A

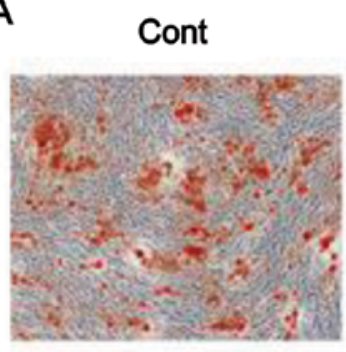

Glucose $16.5 \mathrm{mM}$

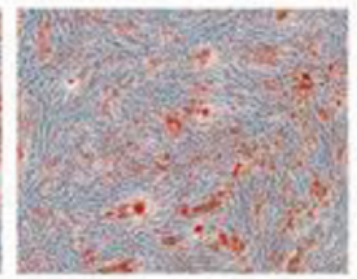

C

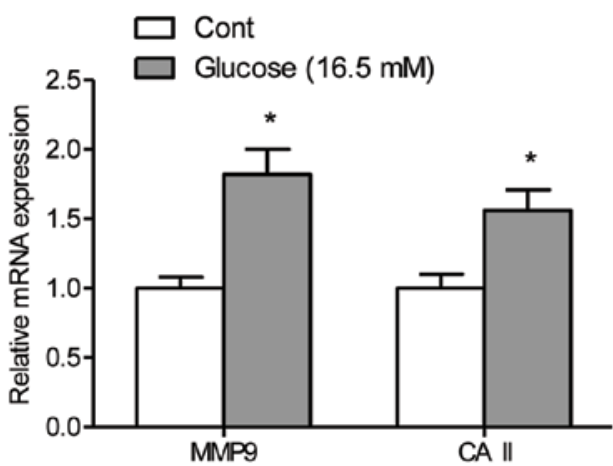

B

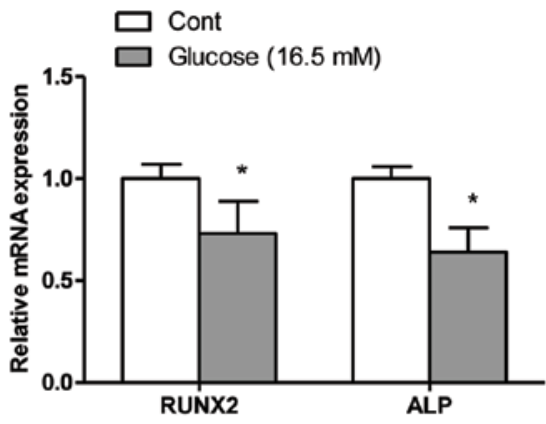

D

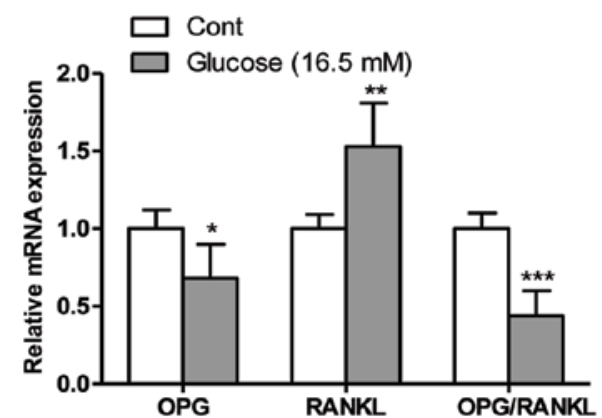

Figure 2. High glucose inhibits osteogenic differentiation. (A) Mineralized matrix formation was assessed by Alizarin Red S staining. mRNA expression levels of (B) the bone formation regulators, runt-related transcription factor (Runx2) and alkaline phosphatase (ALP), and (C) the bone resorption regulators, carbonic anhydrase II (CAII) and matrix metalloproteinase 9 (MMP9) in osteoblasts following exposure to high glucose for 4 days. (D) mRNA expression levels of osteoprotegerin (OPG), receptor activator of nuclear factor- $\kappa \mathrm{B}$ ligand (RANKL) and the ratio of OPG/RANKL. Values are expressed as the means \pm SEM, $\mathrm{n}=3$ parallel experiments in each group. ${ }^{*} \mathrm{P}<0.05,{ }^{* *} \mathrm{P}<0.01$ and ${ }^{* * * *} \mathrm{P}<0.001$ vs. control group (normal glucose).

A

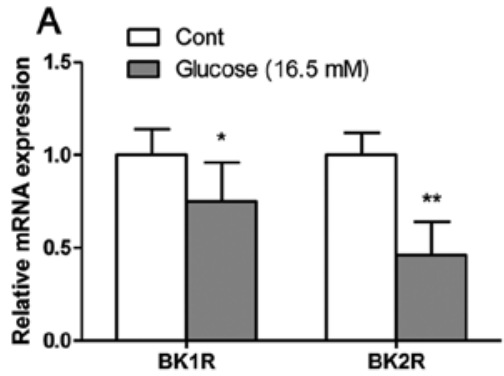

C

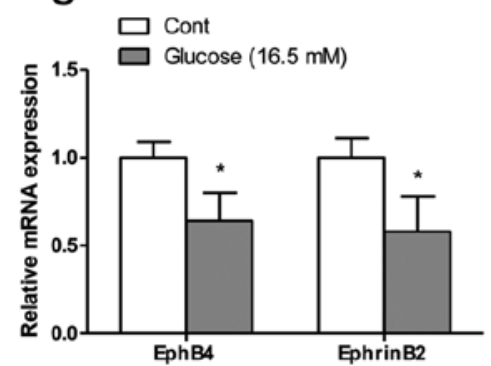

B

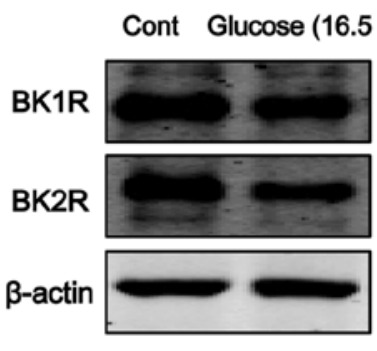

D

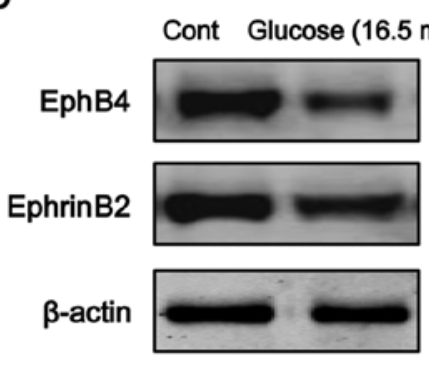

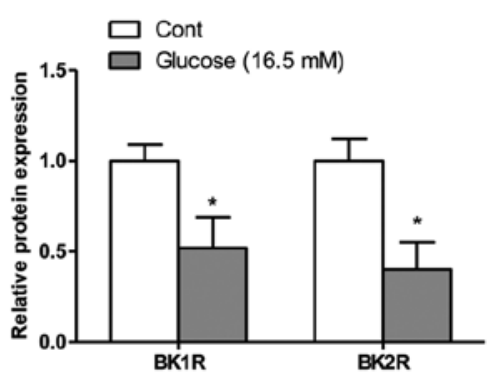

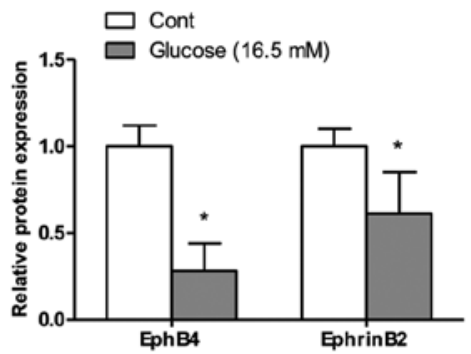

Figure 3. High glucose inhibits the mRNA and protein expression of bradykinin receptors and EphB4/EphrinB2. The mRNA and protein expression levels of (A and B) bradykinin receptors and (C and D) EphB4/EphrinB2 were measured by RT-PCR and western blot analysis, respectively. Values are expressed as the means \pm SEM, $n=3$ parallel experiments in each group. ${ }^{*} \mathrm{P}<0.05$ and ${ }^{* *} \mathrm{P}<0.01$ vs. control group (normal glucose).

High glucose inhibits the $M R N A$ and protein expression of bradykinin receptors and EphB4/EphrinB2. The mRNA and protein expression levels of bradykinin receptors and EphB4/EphrinB2 were measured by RT-PCR and western blot analysis, respectively. Following exposure of the osteo- blasts to high glucose for 4 days, the mRNA (Fig. 3A) and protein (Fig. 3B) expression levels of BK1R and BK2R were significantly decreased compared to the control cells (exposure to normal glucose). Moreover, the mRNA and protein expression levels of EphB4 and EphrinB2 were significantly 
A

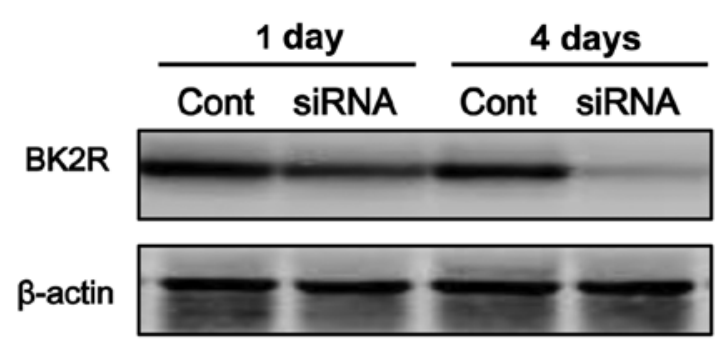

C

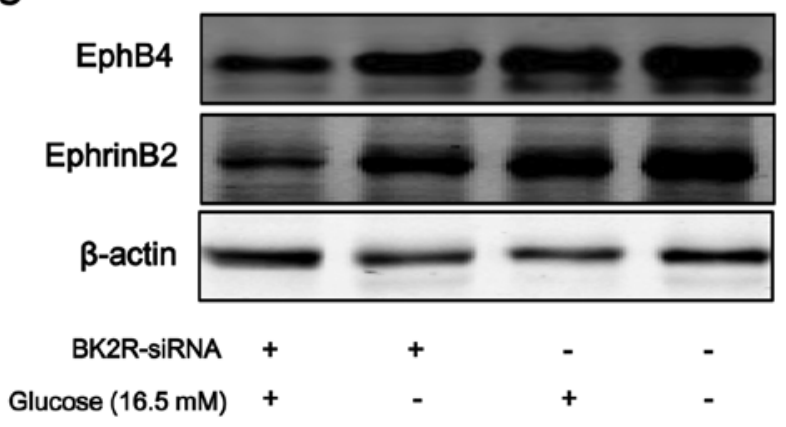

B
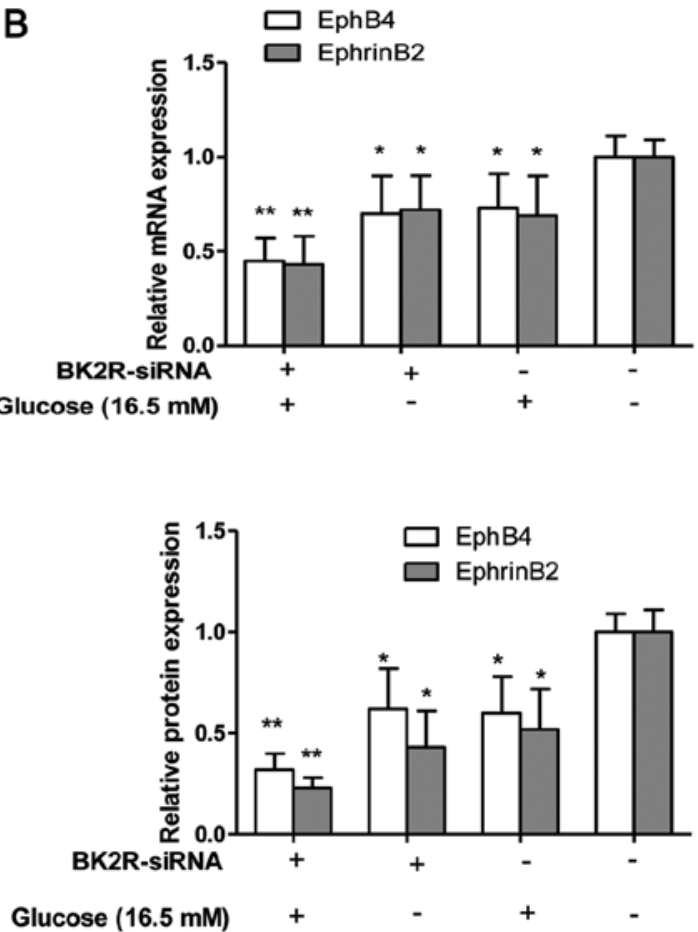

Figure 4. Bradykinin receptor B2 (BK2R) loss-of-function influences the expression of EphB4/EphrinB2. siRNA targeting BK2R was transfected into osteoblasts for 1 and 4 days to suppress the expression of BK2R, which was then measured by (A) western blot analysis. (B) The mRNA and (C) protein expression of EphB4/EphrinB2 was measured when the osteoblasts were transfected with BK2R siRNA. Values are expressed as the means \pm SEM, $n=3$ parallel experiments in each group. ${ }^{*} \mathrm{P}<0.05$ and ${ }^{* *} \mathrm{P}<0.01$, vs. control group.

A

AphB

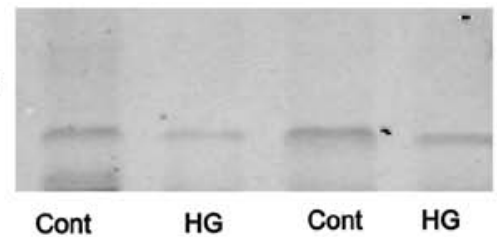

EphrinB2
IP: BK2R

IB: EphB4

IP: BK2R

IB: EphrinB2
B EphB4

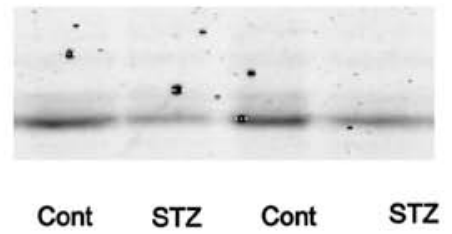

IP: BK2R

IB: EphB4

EphrinB2

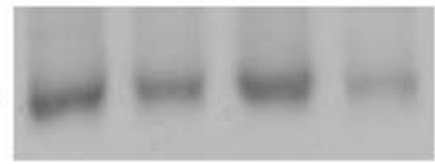

IP: BK2R

IB: EphrinB2

Figure 5. Exposure to high glucose inhibits the interaction between bradykinin receptor B2 (BK2R) and EphB4/EphrinB2. Osteoblasts were incubated with high glucose $(16.5 \mathrm{mM})$ for 4 days. Lysates from (A) osteoblasts and (B) tibias of mice were immunoprecipitated with anti-BK2R antibody. Immunopellets were analyzed by western blot analysis (immunoblotting, IB) using anti-EphB4 or anti-EphrinB2 antibody. IP, immunoprecipitation.

decreased following the exposure of the osteoblasts to high glucose on the fourth day (Fig. 3C and D).

$B K 2 R$ loss-of-function influences the expression of EphB4/EphrinB2. To inhibit the function of BK2R, the osteoblasts were transfected with siRNA against BK2R. Western blot analysis revealed that the protein expression levels of BK2R were markedly decreased following transfection with BK2R siRNA in a time-dependent manner (Fig. 4A). Thus, our data suggested that the siRNA experiments were successfully performed to inhibit the expression of BK2R. BK2R loss-of-function significantly decreased the mRNA $(\mathrm{P}<0.05)$ and protein $(\mathrm{P}<0.05)$ expression of EphB4 and EphrinB2 (Fig. 4B-D). Moreover,
BK2R loss-of-function combined with exposure to high glucose $(16.5 \mathrm{mM})$ markedly decreased the mRNA $(\mathrm{P}<0.05)$ and protein $(\mathrm{P}<0.05)$ expression of EphB4 and EphrinB4 in the osteoblasts compared to the control cells. We found that the mRNA and protein expression levels of both EphB4/EphrinB2 and BK2R, were significantly decreased in the osteoblasts exposed toh high glucose $(16.5 \mathrm{mM})$ in vitro. To examine whether the interaction between EphB4/EphrinB2 and BK2R is dependent on high glucose, cell lysates were prepared from high glucose-exposed cells and immunoprecipitated with antiBK2R antibody. The results revealed that the binding capacity between EphB4/EphrinB2 and BK2R was strong in the control cells, but the binding capacity was weak in the osteoblasts 

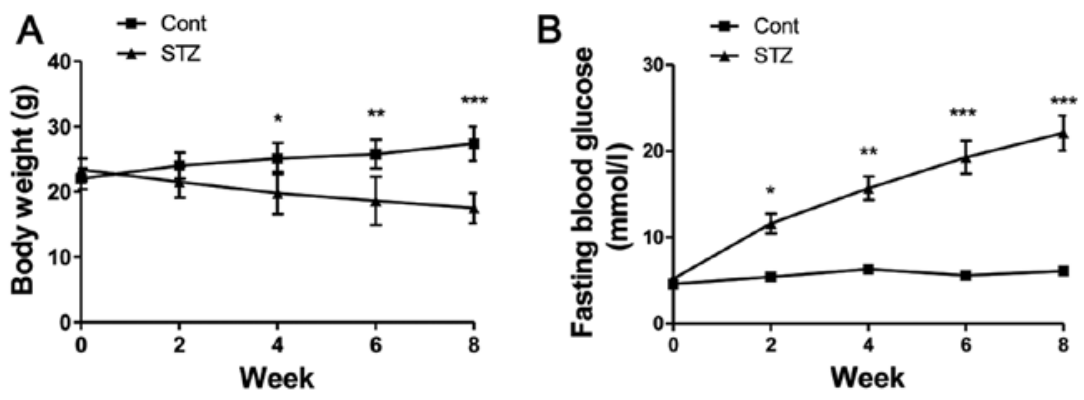

E
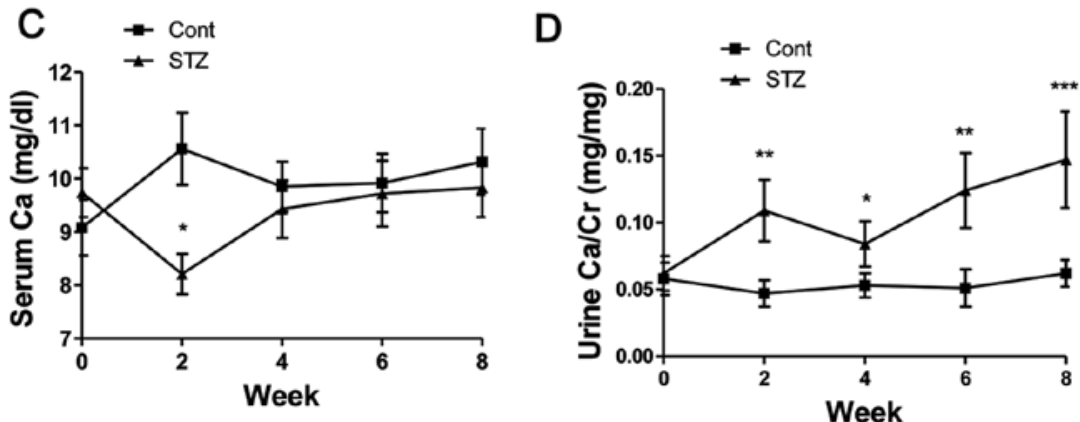

$\mathbf{F}$
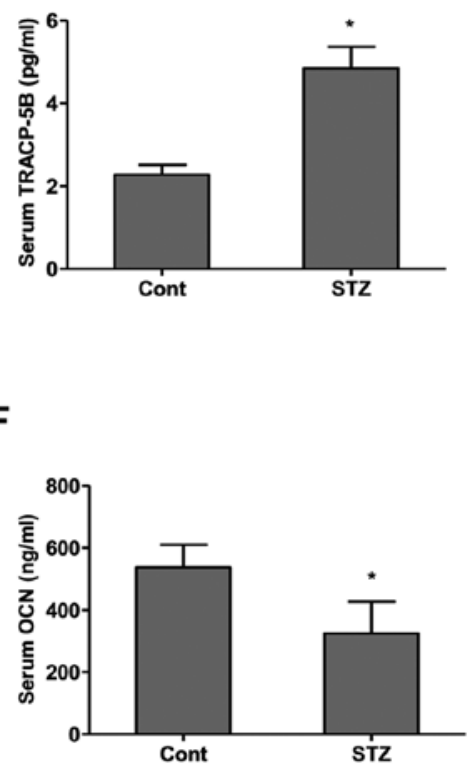

Figure 6. Basic parameters and biomarkers in serum and urine of mice. (A) Body weight (BW), (B) fasting blood glucose (FBG) levels, (C) serum Ca levels and (D) urine Ca levels were recorded every 2 weeks during the experimental period. Serum levels of (E) tartrate resistant acid phosphatase-5b (TRACP-5b) and (F) osteocalcin $(\mathrm{OCN})$ were detected using mouse bioactive enzyme-linked immunosorbent assay (ELISA). Values are expressed as the mean \pm SEM, $\mathrm{n}=6-8 \mathrm{in}$ each group. ${ }^{*} \mathrm{P}<0.05,{ }^{* *} \mathrm{P}<0.01$ and ${ }^{* * *} \mathrm{P}<0.001$ vs. control group (non-diabetic mice).

exposed to high glucose (HG, Fig. 5A). The interaction between EphB4/EphrinB2 and BK2R was also detected in the tibias of mice. The results revealed that the binding capacity between EphB4/EphrinB2 and BK2R was significantly lower in the STZ group compared with the control group (Fig. 5B). These results suggest that the interaction between EphB4/EphrinB2 and BK2R may be involved in STZ-induced diabetes-related osteoporosis.

Basic parameters and biomarkers in serum and urine of mice. The body weights of the non-diabetic control mice steadily increased and the FBG levels were maintained within the normal range of 4-6.1 mmol/1 during the experimental period (Fig. 6A and B). The body weights of the diabetic mice continued to decrease and the FBG levels increased following the administration of STZ (Fig. 6A and B). The FBG levels of the diabetic mice increased from $11.4 \mathrm{mmol} / \mathrm{l}$ at week 2 to $21.6 \mathrm{mmol} / 1$ at week 8 , indicating a significant increase compared to the control group (Fig. 6B). The administration of STZ decreased the serum Ca levels at week $2(\mathrm{P}<0.05)$; however, the serum Ca level showed no obvious difference after week 4 compared to the control group (Fig. 6C). Additionally, our results revealed that the urine Ca level (Fig. 6D) in the STZ group was significantly increased at week $2(\mathrm{P}<0.01)$, week 4 $(\mathrm{P}<0.05)$, week $6(\mathrm{P}<0.01)$ and week $8(\mathrm{P}<0.001)$ compared to the non-diabetic control mice. From these calcium metabolic data, it was demonstrated that hyperglycemia enhanced $\mathrm{Ca}$ excretion in mice with STZ-induced diabetes-related osteoporosis. The serum concentrations of bone turnover markers, such as TRACP-5b, as a bone resorption marker, and OCN, as a bone formation marker, were determined. The results revealed that the serum TRACP-5b levels (Fig. 6E) in the
STZ group were significantly increased, and the serum OCN levels (Fig. 6F) were significantly decreased compared to those of the control group.

Bone histology and TRAP staining. Histological analysis of trabecular bone in the proximal tibia of mice was performed by $H \& E$ staining. The results revealed the increased disconnections and separation among the growth plate and trabecular bone network, as well as the reduction of trabecular bone mass of the primary and secondary spongiosa throughout the proximal tibial epiphysis and proximal tibial metaphysis in the STZ group (Fig. 7A). TRAP staining revealed very few mature osteoclasts in the trabecular bone area below the growth plate in the control group, whereas the number of osteoclasts was significantly increased in this area in the STZ group (Fig. 7B).

Hyperglycemia inhibits the $m R N A$ and protein expression of bradykinin receptors and EphB4/EphrinB2 in tibias of mice. The mRNA and protein expression levels of bradykinin receptors and EphB4/EphrinB2 in the tibias of mice were measured by RT-PCR and western blot analysis, respectively. Eight weeks after the STZ injection, the mRNA (Fig. 8A) and protein (Fig. 8B) expression levels of BK1R and BK2R in the tibias of mice were significantly decreased compared to those of the control group. Moreover, the mRNA and protein expression levels of EphB4 and EphrinB2 were significantly lower in the STZ group than those in the control group (Fig. 8).

\section{Discussion}

In this study, we investigated the physiopathological roles of skeletal EphB4/EphrinB2 and bradykinin receptors in mice 
A

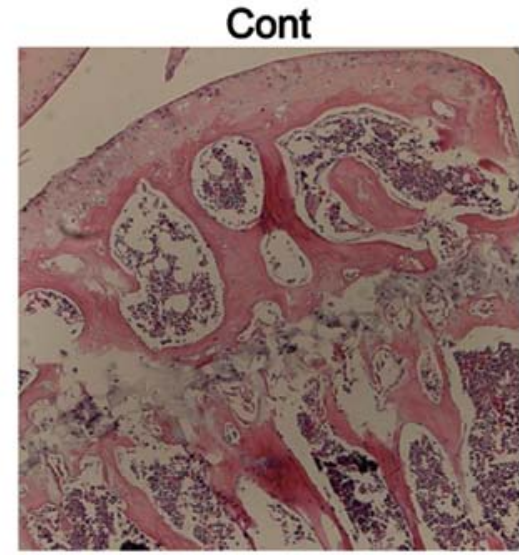

B

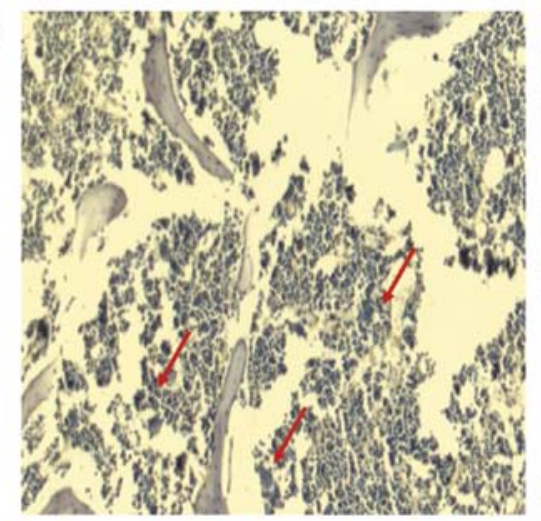

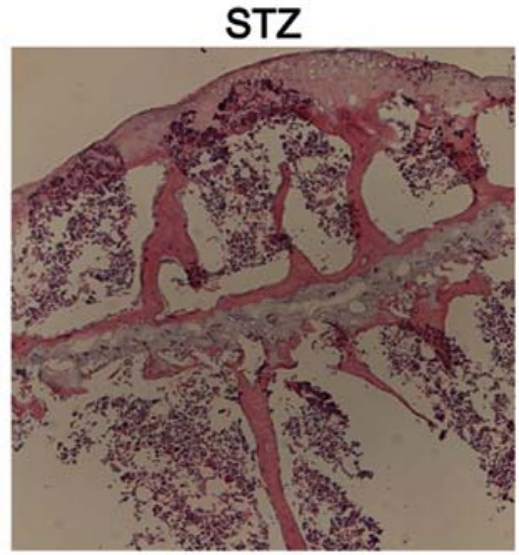

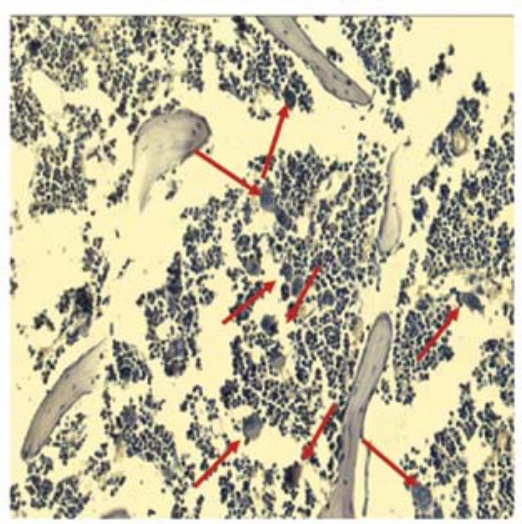

Figure 7. Bone histology and TRAP staining. (A) Hematoxylin and eosin staining (magnification, x100) and (B) TRAP staining (magnification, x200) for osteoclasts (red arrows) in the proximal tibial metaphysis.
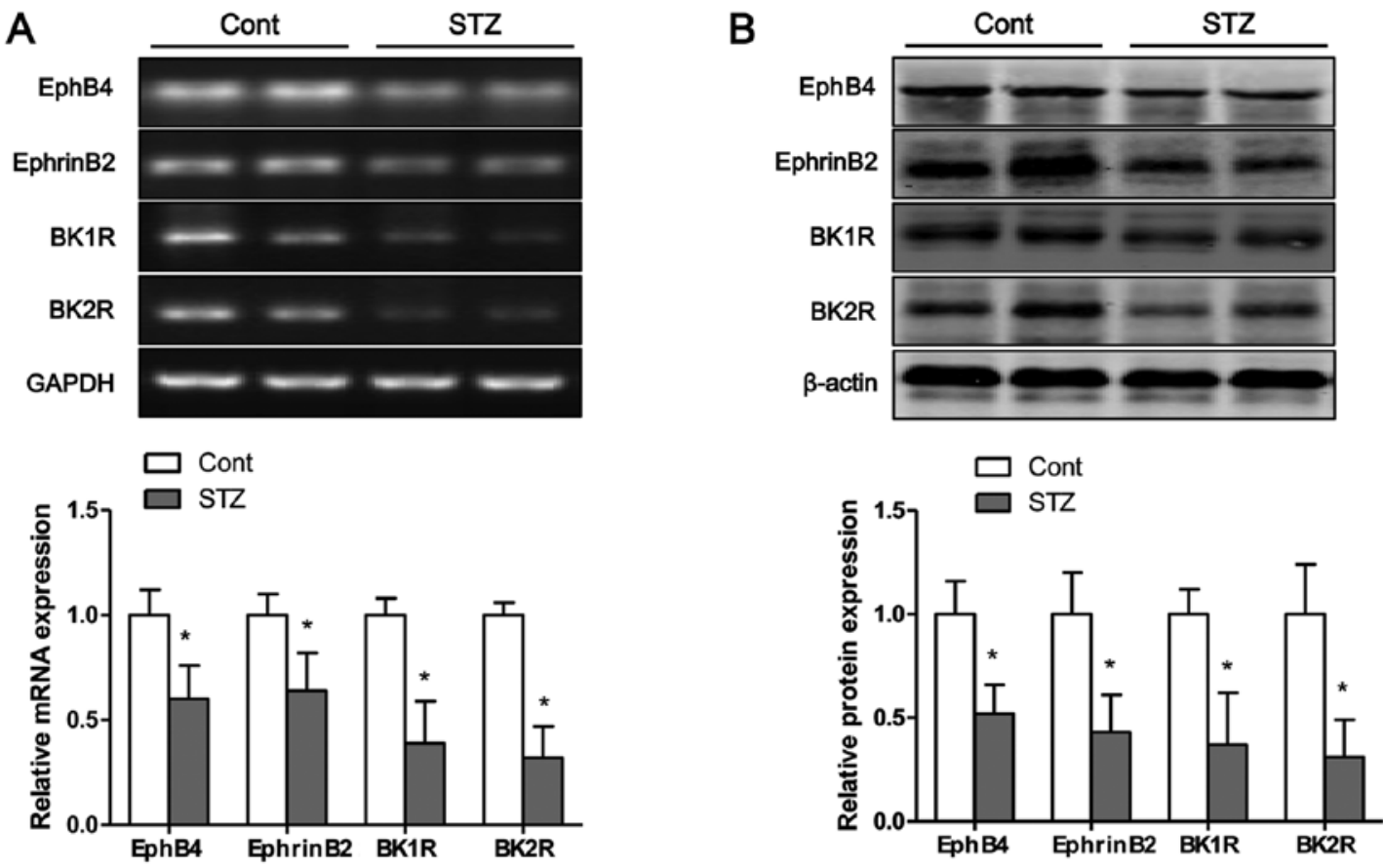

Figure 8. Hyperglycemia inhibits the mRNA and protein expression of bradykinin receptors and EphB4/EphrinB2 in tibias of mice. The (A) mRNA and (B) protein expression of bradykinin receptors and EphB4/EphrinB2 were measured by RT-PCR and western blot analysis, respectively. Values are expressed as the means \pm SEM, $n=6-8$ in each group. ${ }^{*} \mathrm{P}<0.05$ vs. control group.

with diabetes-related osteoporosis. EphB4/EphrinB2 signaling links two major molecular mechanisms of cell differentiation, EphB4 in osteoblasts enhancing osteogenic differentiation and EphrinB2 in osteoclast precursors suppressing osteoclast differentiation, thereby maintaining bone homeostasis (26). In myeloma cells, EphB4/EphrinB2 is expressed at very low 
levels to be detectable, and its activation by EphB4-Fc inhibits myeloma growth and bone disease (29). In the present study, we made several important observations. First, the high glucoseinduced increase in osteoblast apoptosis was confirmed by flow cytometric analysis and the analysis of apoptosis-related protein expression. Next, the EphB4/EphrinB2 axis and bradykinin receptors were found to be dysregulated in osteoblasts following expesure to high glucose, and BK2R loss-of-function downregulated the levels of EphB4/EphrinB2 in vitro. Further experiments demonstrated that high glucose inhibited the interaction between BK2R and EphB4/EphrinB2, which was identified by co-immunoprecipitation. Furthermore, the dysregulated expression of bradykinin receptors and EphB4/EphrinB2 was confirmed in mice with diabetes-related osteoporosis, and the binding capacity between EphB4/EphrinB2 and BK2R was significantly weaker in the mice with diabetes-related osteoporosis than in the non-diabetic control mice.

EphB4/EphrinB2 is a research topic of great scientific value; EphB4/EphrinB2 signaling has been found related with the occurrence of many diseases. The reduced activity of EphB4 has been shown to accelerate the progression of colorectal cancer, and the activation of EphB4 inhibits breast cancer cell growth and migration (30). The communication between osteoclasts and osteoblasts is bidirectional, and forward signaling by EphB4 and possibly other co-expressed Eph receptors promotes the differentiation of osteoblasts, which deposit new bone at sites of resorption by osteoclasts (26). Several Eph receptors present in osteoblasts are regulated by EphrinB reverse signaling, which suppresses osteoblast differentiation through a negative feedback loop that inhibits the expresion of bone sialoprotein $(16,31)$. In this study, we found that exposure to high glucose inhibited osteoblast mineralization and induced bone deterioration, including trabecular bone network degradation and the reduction of the trabecular bone mass of the primary and secondary spongiosa through the suppression of the the expresion of bradykinin recptors and EphB4/EphrinB2 in vitro and in vivo. Previous studies have demonstrated that bradykinin recptors or the EphB4/EphrinB2 signaling pathway maintain bone homeostasis $(13,26)$. In the present study, our data suggested that the interaction between EphB4/EphrinB2 and BK2R plays a vital role in osteogenic differentiation. Moreover, BK2R loss-of-function significantly decreased the mRNA and protein expression levels of EphB4 and EphrinB2.

As previously demonstrated, in Akita diabetic and nondiabetic mice, the lack of both bradykinin receptors results in a severe reduction in bone mineral density, demonstrating the importance of the bradykinin receptors in bone mineralization (14). In this study, the BK1R and BK2R levels were decreased following exposure to high glucose in vitro or downregulated by hyperglycemia in vivo. However, although osteoblasts express both BK1R and BK2R, their stimulation with bradykinin increases the expression of RANKL, which is known to be involved in osteoclastogenesis and to enhance bone resorption (32). The pro-inflammatory cytokines, interleukin (IL)-1 $\beta$ and tumor necrosis factor (TNF)- $\alpha$ can enhance bradykinin receptor expression in osteoblasts, which may help to explain the enhanced bone resorption associated with inflammatory disorders (33). As a key active peptide in the kallikrein-kinin system (KKS), bradykinin can elicit the BK1R and BK2R-mediated activation of ERK1/2 and Akt pathways, which finally leads to the activation of RANKL and decreases the differentiation of osteoblasts with a concomitant increase in osteoclast formation (34). Based on the above-mentioned data, we concluded that bradykinin receptor loss-of-function or gainof-function can influence bone metabolism in vitro and in vivo. Intriguingly, both BK1R and BK2R loss-of-function or BK2R loss-of-function can lead to insulin-resistant or inhibit insulin levels in mice, and B1R and B2R mediate the development of complications in diabetic mice, and the activation of KKS may be beneficial in reducing the severity of complications in diabetic mice $(14,35)$.

In conclusion, in the present study, we demonstrated that high glucose or hyperglycemia inhibited the expression of bradykinin receptors and EphB4/EphrinB2 in vitro or in vivo, respectively. Subsequently, the interaction between bradykinin receptors and EphB4/EphrinB2 was confirmed in osteoblasts, and bradykinin receptor loss-of-function significantly downregulated the levels of EphB4/EphrinB2. These results demonstrated that bradykinin receptors and EphB4/EphrinB2 mediate the development of complications in diabetic mice and suggest that the inactivation of bradykinin receptors and EphB4/EphrinB2 may enhance the severity of complications in mice with diabetes-related osteoporosis.

\section{References}

1. Cummings SR and Melton LJ: Epidemiology and outcomes of osteoporotic fractures. Lancet 359: 1761-1767, 2002.

2. Hamann C, Kirschner S, Günther KP and Hofbauer LC: Bone, sweet bone - osteoporotic fractures in diabetes mellitus. Nat Rev Endocrinol 8: 297-305, 2012.

3. Armas LA, Akhter MP, Drincic A and Recker RR: Trabecular bone histomorphometry in humans with type 1 diabetes mellitus. Bone 50: 91-96, 2012.

4. Loureiro MB, Ururahy MA, Freire-Neto FP, Oliveira GH, Duarte VM, Luchessi AD, Brandão-Neto J, Hirata RD, Hirata MH, Maciel-Neto JJ, et al: Low bone mineral density is associated to poor glycemic control and increased OPG expression in children and adolescents with type 1 diabetes. Diabetes Res Clin Pract 103: 452-457, 2014.

5. Janghorbani M, Feskanich D, Willett WC and Hu F: Prospective study of diabetes and risk of hip fracture: The Nurses' Health Study. Diabetes Care 29: 1573-1578, 2006.

6. Diao TY, Pan H, Gu SS, Chen X, Zhang FY, Wong MS and Zhang Y: Effects of angiotensin-converting enzyme inhibitor, captopril, on bone of mice with streptozotocin-induced type 1 diabetes. J Bone Miner Metab 32: 261-270, 2014.

7. Rao Sirasanagandla S, Ranganath Pai Karkala S, Potu BK and Bhat KM: Beneficial effect of Cissus quadrangularis Linn. on osteopenia associated with streptozotocin-induced type 1 diabetes mellitus in male Wistar rats. Adv Pharmacol Sci 2014: 483051, 2014.

8. Fu C, Zhang X, Ye F and Yang J: High insulin levels in KK-Ay diabetic mice cause increased cortical bone mass and impaired trabecular micro-structure. Int J Mol Sci 16: 8213-8226, 2015.

9. Guan CC, Yan M, Jiang XQ, Zhang P, Zhang XL, Li J, Ye DX and Zhang FQ: Sonic hedgehog alleviates the inhibitory effects of high glucose on the osteoblastic differentiation of bone marrow stromal cells. Bone 45: 1146-1152, 2009.

10. Wittrant Y, Gorin Y, Woodruff K, Horn D, Abboud HE, Mohan S and Abboud-Werner SL: High d(+)glucose concentration inhibits RANKL-induced osteoclastogenesis. Bone 42: 1122-1130, 2008.

11. Irie N, Takada Y, Watanabe Y, Matsuzaki Y, Naruse C, Asano M, Iwakura Y, Suda T and Matsuo K: Bidirectional signaling through ephrinA2-EphA2 enhances osteoclastogenesis and suppresses osteoblastogenesis. J Biol Chem 284: 14637-14644, 2009.

12. Matsuo $\mathrm{K}$ and Otaki N: Bone cell interactions through Eph/ephrin: Bone modeling, remodeling and associated diseases. Cell Adhes Migr 6: 148-156, 2012.

13. Kwan Tat S, Pelletier JP, Amiable N, Boileau C, Lavigne M and Martel-Pelletier J: Treatment with ephrin B2 positively impacts the abnormal metabolism of human osteoarthritic chondrocytes. Arthritis Res Ther 11: R119, 2009. 
14. Cheng S, Zhao SL, Nelson B, Kesavan C, Qin X, Wergedal J, Mohan S and Xing W: Targeted disruption of ephrin B1 in cells of myeloid lineage increases osteoclast differentiation and bone resorption in mice. PLoS One 7: e32887, 2012.

15. Arthur A, Zannettino A, Panagopoulos R, Koblar SA, Sims NA, Stylianou C, Matsuo K and Gronthos S: EphB/ephrin-B interactions mediate human MSC attachment, migration and osteochondral differentiation. Bone 48: 533-542, 2011.

16. Watanabe T, Sato Y, Saito D, Tadokoro R and Takahashi Y: EphrinB2 coordinates the formation of a morphological boundary and cell epithelialization during somite segmentation. Proc Natl Acad Sci USA 106: 7467-7472, 2009.

17. Xing W, Kim J, Wergedal J, Chen ST and Mohan S: Ephrin B1 regulates bone marrow stromal cell differentiation and bone formation by influencing TAZ transactivation via complex formation with NHERF1. Mol Cell Biol 30: 711-721, 2010.

18. Davy A, Aubin J and Soriano P: Ephrin-B1 forward and reverse signaling are required during mouse development. Genes Dev 18: 572-583, 2004.

19. Davy A, Bush JO and Soriano P: Inhibition of gap junction communication at ectopic Eph/ephrin boundaries underlies craniofrontonasal syndrome. PLoS Biol 4: e315, 2006.

20. Stiffel V, Amoui M, Sheng MH, Mohan S and Lau KH: EphA4 receptor is a novel negative regulator of osteoclast activity. J Bone Miner Res 29: 804-819, 2014.

21. Yamada T, Yuasa M, Masaoka T, Taniyama T, Maehara H, Torigoe I, Yoshii T, Shinomiya K, Okawa A and Sotome S: After repeated division, bone marrow stromal cells express inhibitory factors with osteogenic capabilities, and EphA5 is a primary candidate. Bone 57: 343-354, 2013.

22. Zhao C, Irie N, Takada Y, Shimoda K, Miyamoto T, Nishiwaki T, Suda T and Matsuo K: Bidirectional ephrinB2-EphB4 signaling controls bone homeostasis. Cell Metab 4: 111-121, 2006.

23. Campbell DJ: The renin-angiotensin and the kallikrein-kinin systems. Int J Biochem Cell Biol 35: 784-791, 2003.

24. Campbell DJ: The kallikrein-kinin system in humans. Clin Exp Pharmacol Physiol 28: 1060-1065, 2001.

25. Gonçalves-Zillo TO, Pugliese LS, Sales VM, Mori MA, Squaiella-Baptistão CC, Longo-Maugéri IM, Lopes JD, de Oliveira SM, Monteiro AC and Pesquero JB: Increased bone loss and amount of osteoclasts in kinin B1 receptor knockout mice. J Clin Periodontol 40: 653-660, 2013.
26. Kakoki M, Sullivan KA, Backus C, Hayes JM, Oh SS, Hua K, Gasim AM, Tomita H, Grant R, Nossov SB, et al: Lack of both bradykinin $\mathrm{B} 1$ and $\mathrm{B} 2$ receptors enhances nephropathy, neuropathy, and bone mineral loss in Akita diabetic mice. Proc Natl Acad Sci USA 107: 10190-10195, 2010.

27. Tsukamoto T, Li X, Morita H, Minowa T, Aizawa T, Hanagata N and Demura M: Role of S-palmitoylation on IFITM5 for the interaction with FKBP11 in osteoblast cells. PLoS One 8: e75831, 2013.

28. Bi Y, Nielsen KL, Kilts TM, Yoon A, A Karsdal M, Wimer HF, Greenfield EM, Heegaard AM and Young MF: Biglycan deficiency increases osteoclast differentiation and activity due to defective osteoblasts. Bone 38: 778-786, 2006.

29. Pennisi A, Ling W, Li X, Khan S, Shaughnessy JD Jr, Barlogie B and Yaccoby S: The ephrinB2/EphB4 axis is dysregulated in osteoprogenitors from myeloma patients and its activation affects myeloma bone disease and tumor growth. Blood 114: 1803-1812, 2009.

30. Pasquale EB: Eph-ephrin bidirectional signaling in physiology and disease. Cell 133: 38-52, 2008.

31. Shimizu E, Tamasi J and Partridge NC: Alendronate affects osteoblast functions by crosstalk through EphrinB1-EphB. J Dent Res 91: 268-274, 2012.

32. Brechter AB and Lerner UH: Bradykinin potentiates cytokineinduced prostaglandin biosynthesis in osteoblasts by enhanced expression of cyclooxygenase 2, resulting in increased RANKL expression. Arthritis Rheum 56: 910-923, 2007.

33. Brechter AB, Persson E, Lundgren I and Lerner UH: Kinin B1 and $\mathrm{B} 2$ receptor expression in osteoblasts and fibroblasts is enhanced by interleukin- 1 and tumour necrosis factor-alpha. Effects dependent on activation of NF-kappaB and MAP kinases. Bone 43: 72-83, 2008.

34. Srivastava S, Sharma K, Kumar N and Roy P: Bradykinin regulates osteoblast differentiation by Akt/ERK/NFkB signaling axis. J Cell Physiol 229: 2088-2105, 2014.

35. Duka I, Shenouda S, Johns C, Kintsurashvili E, Gavras I and Gavras H: Role of the $\mathrm{B}(2)$ receptor of bradykinin in insulin sensitivity. Hypertension 38: 1355-1360, 2001. 